\title{
Traumatismo Toracoabdominal Penetrante: Que Cavidad Operar Primero?
}

\author{
Celeste Echavarria ${ }^{1}$, Sofia Bou ${ }^{2}$, Favio Guzman ${ }^{3}$, Cristian Assell $^{4}$, Juliana Nazaretto ${ }^{5}$, Andrea Potes ${ }^{6}$, Guillermo Barillaro ${ }^{7}$
}

\begin{abstract}
Resumen
Aim y antecedentes: Los pacientes con traumatismos toracoabdominales penetrantes (TTAP) por arma de fuego (HAF) o por arma cortopunzante (HCP) plantean el desafío de decidir que cavidad operar prioritariamente. Iniciar la cirugía en la cavidad con las lesiones menos graves puede retrasar el manejo de una hemorragia o de un taponamiento cardiaco letales.

Objetivo: El objetivo de este trabajo fue el análisis de:

- Características de los pacientes con TTAP con secuencia de intervenciones quirúrgicas combinadas.

- Características del error por inapropiada secuencia de dichos procedimientos operatorios.

- Relación de esos factores anteriores con la mortalidad.

Diseño: Retrospectivo observacional.

Material y metodo: Revisión de las historias clínicas de los pacientes asistidos en nuestra institución entre enero del 2005 y diciembre del 2018 , con TTAP que requirieron procedimientos operatorios tanto en el tórax (drenaje pleural o toracotomía) como en el abdomen (laparotomía o laparoscopia).

Resultado: Fueron asistidos 79 pacientes con TTAP, 48 con normalidad hemodinámica (grupo l) y 31 con shock hipovolémico (grupo II). En el grupo I predominaron las HCP (40) sobre HAF (8), y las lesiones del lado izquierdo (42) (87,5\%). En este grupo no hubo errores en el manejo quirúrgico secuencial ni se registró mortalidad. En el grupo II predominaron las HAF transfixiantes de la línea media. En 8 casos se registró un error al abordar primero la cavidad con lesiones menos graves (en 4 casos el tórax y en 4 casos el abdomen), falleciendo 7 de los mismos y determinando una mortalidad de $25,8 \%$ para el grupo II. El análisis del error en esos 8 casos hallo que en 5 fue potencialmente prevenible y que estuvo relacionado con resultados erróneos de la ecografía y/o subestimación del débito del drenaje pleural y de la radiografía de tórax post-drenaje pleural.

Conclusione: Los pacientes con TTAP y hemodinámicamente compensados presentaron predominio de HCP del lado izquierdo y no tuvieron errores en el manejo secuencial de cavidades ni mortalidad. En cambio en aquellos con TTAP y shock, predominaron las HAF y los trayectos transfixiantes de la línea media, y este grupo fue el que tuvo exclusivamente los errores de manejo y la mortalidad. La mayoría de los errores en el manejo fueron considerados potencialmente prevenibles dado que se relacionaron con resultados falsos de la ecografía y con subestimación del débito del drenaje pleural y de la radiografía de tórax post-drenaje pleural.
\end{abstract}

Keywords: Análisis de mortalidad, Calidad en trauma, Decision making, Trauma de torax abierto, Trauma penetrante de abdomen, Ultrasonografía.

\section{Abstract}

Background: Patients with penetrating thoracoabdominal trauma (PTAT) by gunshot wounds (GSW) or by stab wounds (SW) pose the challenge of deciding which cavity to operate first. Initiating surgery in the cavity with less severe injuries may delay the management of a fatal hemorrhage or cardiac tamponade.

Aim and objective: The objective of this work was the analysis of:

- Characteristics of patients with PTAT with a sequence of combined surgical interventions.

- Characteristics of the error due to inappropriate sequence of said operative procedures.

- Relationship of these previous factors with mortality.

Design: Observational retrospective.

Materials and methods: Review of the medical records of the patients assisted in our institution between January 2005 and December 2018 , with PTAT that required operative procedures both in the chest (pleural drainage or thoracotomy) and in the abdomen (laparotomy or laparoscopy). Results: Seventy-nine patients with PTAT, 48 with normal hemodynamics (group I) and 31 with hypovolemic shock (group II) were assisted. In group I, SW (40) predominated over GSW (8), and lesions on the left side (42) (87.5\%). In this group, there were no errors in sequential surgical management and no mortality was recorded. In group II, transfixing GSW of the midline predominated. In eight cases, an error was recorded when first approaching the cavity with less serious injuries (in four cases the thorax and in four cases the abdomen), seven of them died and determining mortality of $25.8 \%$ for group II. The analysis of the error in these eight cases found that in five it was potentially preventable and that it was related to erroneous results of the ultrasound and/or underestimation of the output of the pleural drainage and of the post-pleural drainage chest radiograph. Conclusion: Patients with PTAT and hemodynamically compensated presented a predominance of left-sided SW and had no errors in the sequential management of cavities or mortality. On the other hand, in those with PTAT and shock, GSW and transfixing paths of the midline predominated, and this group was the one that had exclusively the handling errors and mortality. Most of the errors in management were considered potentially preventable since they were related to false results of the ultrasound and underestimation of the output of the pleural drainage and of the post-pleural drainage chest radiograph.

Keywords: Abdominal penetrating trauma, Decision making, Mortality analysis, Open thoracic trauma, Quality in trauma management, Ultrasonography

Panamerican Journal of Trauma, Critical Care \& Emergency Surgery (2021): 10.5005/jp-journals-10030-1318

(c) The Author(s). 2021 Open Access This article is distributed under the terms of the Creative Commons Attribution 4.0 International License (https:// creativecommons.org/licenses/by-nc/4.0/), which permits unrestricted use, distribution, and non-commercial reproduction in any medium, provided you give appropriate credit to the original author(s) and the source, provide a link to the Creative Commons license, and indicate if changes were made. The Creative Commons Public Domain Dedication waiver (http://creativecommons.org/publicdomain/zero/1.0/) applies to the data made available in this article, unless otherwise stated. 


\section{INTRODUCCIÓN}

Los pacientes con traumatismos toracoabdominales penetrantes (TTAP) por arma de fuego (HAF) o arma cortopunzante (HCP) y con lesiones potenciales tanto en tórax como en abdomen, plantean el desafío de decidir que cavidad operar prioritariamente. Iniciar la cirugía en la cavidad con las lesiones menos graves puede retrasar el manejo de una hemorragia o taponamiento cardiaco letales.

En un escenario de shock hipovolémico agregado, la evaluación clínica más dificultosa y la necesidad de tomar rápidamente conductas aumentan las posibilidades de error al decidir que cavidad operar primero.

El objetivo de este trabajo fue:

- Analizar las características de los pacientes con TTAP con secuencia de intervenciones quirúrgicas combinadas en tórax y abdomen.

- La incidencia de error por inapropiada secuencia de procedimientos operatorios y las causas de dichos errores.

- La relación de esos factores anteriores con la mortalidad.

\section{Método de Investigación}

Con método retrospectivo observacional se revisaron las historias clínicas de los pacientes asistidos en nuestra institución, entre enero del 2005 y diciembre del 2018, con TTAP que requirieron los siguientes procedimientos operatorios: drenaje pleural o toracotomía el tórax, así como laparotomía o videolaparoscopia en el abdomen.

La región toracoabdominal fue definida en sus tres zonas: anterior, lateral y posterior. En la zona anterior por una línea imaginaria bimamilar anterior a nivel del cuarto espacio intercostal como límite superior, y por el reborde costal como límite inferior. En la zona lateral entre las líneas axilares anterior y posterior, con límite superior es el sexto espacio intercostal y límite inferior en el reborde costal. Y en la zona posterior con límite superior en un trazo que une las puntas escapulares a la altura del séptimo espacio intercostal y con límite inferior dado nuevamente por el reborde costal.

En la asistencia de este grupo de traumatizados se consideró error a aquella situación en la cual se abordó en primer término a la cavidad con una hemorragia o lesión menos grave o de menor letalidad al compararla con otra lesión presente y concomitante en la otra cavidad.

Con respecto a las definiciones del tipo de muerte en base a su posibilidad de prevención, se consideró como muerte no prevenible a aquella provocada por lesiones fatales pese a tener cuidados óptimos y con posibilidad de supervivencia estimada mediante la metodología TRISS menor al 25\%. Una muerte potencialmente prevenible se definió como aquella provocada por una lesión considerada muy severa pero sobrevivible bajo condiciones óptimas; en estos casos la probable supervivencia fue calculada entre el 25 y 50\%. Y la definición de una muerte prevenible incluyo a aquellas provocadas por lesiones consideradas sobrevivibles y con una sospecha sobre la calidad de su atención; en estos casos la probable supervivencia fue calculada como superior al 50\%.

Fueron asistidos 79 pacientes con TTAP, los cuales en su ingreso recibieron la atención en base a las normativas del curso ATLS. Según su condición hemodinámica los casos fueron divididos en dos grupos: grupo I (compensados o normales) y grupo II (descompensados o anormales o en shock).
${ }^{1-7}$ Emergency Department, Hospital Interzonal General de Agudos (HIGA) “Dr Oscar Alende", Ciudad de Mar del Plata, Argentina

Corresponding Author: Guillermo Barillaro, Emergency Department, Hospital Interzonal General de Agudos (HIGA) "Dr Oscar Alende", Ciudad de Mar del Plata, Argentina, e-mail: barillaroguillermo@gmail.com

How to cite this article: Echavarria C, Bou S, Guzman F, et al. Traumatismo Toracoabdominal Penetrante: Que Cavidad Operar Primero?. Panam J Trauma Crit Care Emerg Surg 2021;10(2):71-77.

Source of support: Nil

Conflict of interest: None

La división de la serie en dos grupos de pacientes en base a la condición hemodinámica de los mismos obedeció al reconocimiento de que ese estado es considerado en todos los algoritmos como una piedra angular para el manejo de los traumatismos penetrantes. Dicho estado condiciona el tipo de estudios complementarios que esos pacientes pueden o no recibir, así como la indicación del tratamiento operatorio.

La definición de anormalidad hemodinámica correspondió a pacientes con una tensión arterial sistólica inferior a $90 \mathrm{~mm} \mathrm{Hg}$ y con grados estimados de pérdidas hemorrágicas de tipo III o IV según la clasificación del curso ATLS@. ${ }^{1}$

Se realizo análisis con STATA 13, se eligió un nivel de significación estadística alfa de 0,01 . Se utilizo cómo test estadístico chi2 y test exacto de Fisher.

\section{Resultados}

El grupo I incluyo 48 (60.7\%) pacientes con normalidad hemodinámica, mientras que en el grupo Il se registraron 31 (39.3\%) pacientes con condición de shock.

En el grupo I, con 48 pacientes, predominaron las HCP (40) sobre HAF (8), y las lesiones del lado izquierdo de la zona toracoabdominal (42) sobre las del lado derecho (6). En todos los pacientes de este grupo se realizaron ecografía de tórax y de abdomen (a cargo de departamento de Diagnostico por imágenes) así como radiografía de tórax en la sala de emergencias, y en todos ellos (48) se practicó un drenaje pleural en esa misma sala siguiendo las guías del curso ATLS. En los 6 casos de heridas toracoabdominales derechas pudo realizarse, luego del drenaje pleural, un tratamiento no operatorio de una lesión hepática con buena evolución, luego de agregar una tomografía toracoabdominal con contraste endovenoso (TAC) a los estudios por imágenes previos. Dentro de los 42 casos de heridas del lado izquierdo, en un subgrupo de 14 pacientes se indicó una laparotomía exploradora ante la presencia de peritonitis o de evisceración; en los 28 casos restantes de las heridas del lado izquierdo se realizó una videolaparoscopia diagnóstica para descartar una lesión diafragmática, de acuerdo con un protocolo previo de nuestra institución. Esas laparoscopias hallaron laceraciones diafragmáticas en 14 casos (50\%), mientras que fueron negativas en la mitad restante.

En este grupo I no se registró error en la secuencia de prioridad elegida para el abordaje de la cavidades afectadas, así como tampoco hubo mortalidad entre estos traumatizados.

El grupo Il incluyo 31 casos de pacientes con descompensación hemodinámica en su ingreso. En ellos predominó el mecanismo de HAF (16) sobre el de las HCP (15), así como el trayecto transfixiante (12) sobre la ubicación del lado izquierdo (11) o derecho (8). En todos los pacientes de este grupo se realizó ecografía de tórax y de 
abdomen (a cargo de departamento de Diagnostico por imágenes), mientras que la radiografía de tórax fue practicada en 20 (64.5\%) de los casos de este grupo (Fig. 1).

En 27 (87.1\%) pacientes de este grupo se practicó un drenaje pleural en la sala de shock siguiendo las guías del curso ATLS, mientras que en los 4 casos restantes se procedió directamente a una toracotomía de reanimación en la sala de shock, por condición de parada cardiaca inminente y de acuerdo con un protocolo previo de nuestra institución.

En 15 casos la cavidad abordada inicialmente fue el abdomen a través de una laparotomía mediana suprainfraumbilical; en los restantes 16 casos la cavidad abordada inicialmente fue el tórax a través de incisiones anterolaterales (derecha, izquierda, o bilateral tipo clamshell).

En este grupo II se registraron los 8 casos (25.8\%) de error al elegir que cavidad abordar primero. Se consideró una secuencia incorrecta de acción en esos casos, en los cuales la cavidad abordada en primer término no presentaba la lesión más grave o la mayor hemorragia, al compararla con la otra cavidad que presentaba una lesión concomitante y más severa. En 4 de ellos se realizó inicialmente una laparotomía, cuando la lesión más grave en realidad se hallaba en el tórax; se trataba de una herida cardiaca en dos casos, una laceración en la aorta torácica descendente en otro y una lesión de arteria intercostal en el restante. En estos pacientes los factores relacionados con el error incluyeron dos casos de sangrado transdiafragmatico central desde heridas cardiacas hacia la cavidad peritoneal y dos casos en los cuales se subestimo un drenaje pleural con un debito de $1200 \mathrm{cc}$.

En los otros 4 casos de error al elegir la secuencia de abordaje se trató de casos en los cuales se realizó inicialmente una toracotomía, cuando la lesión más grave se hallaba en el abdomen; se trató de 3 casos de lesión de vena cava inferior retrohepática y de un caso de lesión hepática. En estos pacientes los factores relacionados con el error incluyeron tres resultados falsos de la ecografía y un caso de lesión diafragmática asociada donde el sangrado pasaba desde una lesión de vena cava retrohepática hacia el tórax. Los resultados falsos de la ecografía comprendieron dos casos de falso positivo informando liquido en saco pericárdico y un caso falso negativo que omitió la presencia de líquido intra-abdominal (Figs 2 y 3 ).

En este grupo II, con 31 pacientes, hubo 12 (38.7\%) fallecidos, lo cual constituyó también la mortalidad de la serie completa (12/79) (15.2\%), dado que en el grupo I no hubo decesos. Entre esos 12 fallecidos, 9 habían sufrido HAF y 8 tenían trayectorias tansfixiantes de la línea media.

En este grupo II también se halló que entre los 23 pacientes que no presentaron error en la secuencia de abordaje la mortalidad

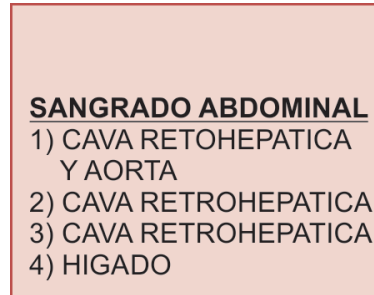

Fig. 1: Casos con error en la secuencia operatoria y su valoración del nivel de prevenibilidad


SANGRADO TORACICO

1) CARDIACO

2) A. INTERCOSTAL

3) CARDIACO

4) AORTA TORACICA DESCENDENTE

SANGRADO TORACICO

1) SANGRADO

TRANSDIAFRAG

MATICO

2) SUBESTIMACION

DREN PLEURAL

1200CC FALTA RX TX

POST DREN

3) SANGRDO

TRANSDIAFRAG MATICO

4) SUBESTIMACION

DREN PLEURAL

1200CC FALTA FALTA RX

TX POST DREN

Fig. 2: Casos en los cuales hubo errores en la secuencia operatoria 

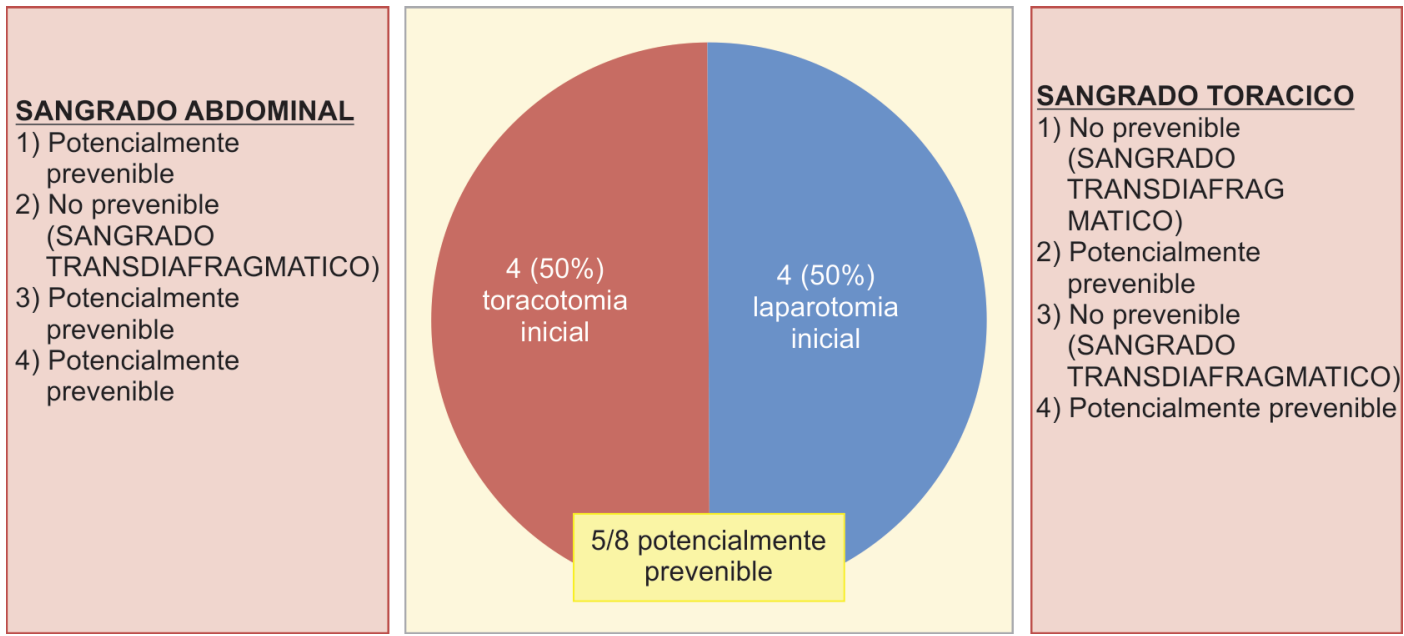

Fig. 3: Causas de los errores en los casos con secuencia operatoria inapropiada

fue de $21.7 \%$ (5 casos), tres de ellos a raíz de falla orgánica múltiple postoperatoria y dos por hemorragia intraoperatoria. Por otro lado entre quienes sí tuvieron error en la secuencia de abordaje, 8 casos, la mortalidad fue mayor con una incidencia de $87.5 \%$ (7 de esos 8 pacientes); la mayoría de estos fallecimientos se debieron a hemorragia intraoperatoria (6 de 7).

En este segundo grupo la anormalidad hemodinámica por la condición de shock hipovolémico, el predominio del mecanismo de HAF y el error de secuencia de abordaje (factores todos ellos no presentes en el primer grupo) se asociaron a una mayor mortalidad.

Con respecto a las asociaciones estadisticas halladas, se puede mencionar:

- La mortalidad en el grupo Il fue de $38 \%$ mientras que en el grupo I fue de $0 \%$, con una $p<0.001$.

- En el grupo I los pacientes con lesiones en el lado izquierdo fueron 42 (87.5) requiriendo todos ellos cirugía, a diferencia de los del lado derechos, con una $p<0.001$.

- Los pacientes con HAF tuvieron mayor incidencia de inestabilidad hemodinámica, o sea, de pertenecer al grupo II, $66 \%$ (16 casos) vs $33 \%$ ( 8 casos) del grupo I, con a $p=0.002$.

- Dentro del grupo en shock, donde se presentaron los pacientes con error de secuencia de cavidad operada, los mismos tuvieron una mortalidad elevada, siendo del $87.5 \%(p=0.002)$.

- Los pacientes con HAF tuvieron una mortalidad del $37.5 \%$ vs $5 \%$ de mortalidad en HCP, con una $p=0.001$

- Dentro del grupo en shock, en los pacientes en quienes hubo error en secuencia de cavidad operada primero, hubo 50\% de resultados falsos en la ecografía incial, con un $p=0002$.

\section{Discusión}

Los pacientes con traumatismos toracoabdominales penetrantes (TTAP) por arma de fuego o arma corto punzante y con lesiones potenciales tanto en tórax como en abdomen, plantean el desafío de decidir que cavidad operar de modo prioritario. ${ }^{2,3}$

El abordaje inicial de la cavidad menos afectada puede asociarse a un aumento de la morbimortalidad por retraso del tratamiento de la lesión más grave.

Estadísticamente ha sido reportado que dos tercios de estos pacientes suelen ser manejados con drenajes pleurales y un abordaje abdominal (laparotomía o laparoscopía), estando ellos compensados hemodinamicamente. ${ }^{4-13}$ Por otro lado, el tercio restante suele requerir laparotomía y toracotomía a raíz de lesiones multicavitarias. En este grupo predominan las heridas por arma de fuego y la condición de shock hipovolémico, lo cual transforma a este grupo en el más problemático a la hora de su manejo.

El abordaje quirúrgico de al TTAP se enfrenta con varios desafíos: más de una fuente de sangrado, más de una cavidad abierta, peor condición global, y si la bala atravesó la línea media un aumento considerable de la mortalidad..$^{14}$

Ha sido descripto hasta un 25 a 30\% de error en la decisión de que cavidad operar primero. Los factores causales investigados incluyeron no considerar trayectoria de la bala, inestabilidad hemodinámica que impide realizar estudios completos, valoración incorrecta del débito del drenaje pleural (ocluido, doblado, o drenando hemorragia abdominal) y no haber descartado un taponamiento cardiaco presente. ${ }^{15}$

Otros autores han comunicado una incidencia de 31 a 39\% de exploración inicial errónea cuando se realizaron procedimientos combinados, y un aumento de la mortalidad (31 a 59\%) cuando se realizan tales abordajes combinados. ${ }^{16}$ En caso de exploración inicial errónea el porcentaje fue mayor cuando la exploración se inició en el abdomen ( 53 vs $36 \%$ ) y la mortalidad aumentó a un $69 \%$ en caso del cruce de la línea media torácica por el proyectil.

En base a esas dificultades y riesgos descriptos en el manejo de este tipo de traumatizados, algunos autores han propuesto adoptar una serie de precauciones, tanto preoperatorias como intraoperatorias, con el fin de disminuir la incidencia del error en el abordaje y de la mortalidad. Esas medidas han incluido los siguientes recursos: ${ }^{14}$

\section{En Fase Preoperatoria}

- Uso liberal del drenaje pleural, con bajo umbral para su colocación ante la asociación de hemoneumotórax y condición de shock.

- Reevaluación del débito del drenaje pleural y de la radiografía de tórax post-drenaje para descartar la presencia de un hemotórax retenido.

- Considerar que un debito del drenaje pleural superior a 1000 cc. luego de un traumatismo penetrante se asocia fuertemente a la necesidad de toracotomía mayor para el tratamiento de lesiones hemorrágicas. 
- Considerar que un hemotórax retenido puede ocultar una importante fuente de sangrado (arteria intercostal, mamaria, aorta, corazón).

- Utilizar en forma sistemática en la sala de shock a la ecografía en su modo extendido, incluyendo la valoración del saco pericárdico y de la cavidad pleural. ${ }^{17-19}$

\section{En fase intraoperatoria:}

- Ante una lesión por HAF que atraviesa línea media: pensar en necesidad de una cirugía de control del daño

- Mantener flexibilidad táctica para agregar otro abordaje cuando se está trabajando en la cavidad opuesta

- Buscar claves de que algo está ocurriendo al otro lado del diafragma a través del diálogo con el anestesista y ante ciertas situaciones: hipotensión inexplicada, falta de respuesta a la reanimación con fluidos o sangre, aumento en la presión de la vía aérea (¿hemoneumotórax?) o aumento en la presión venosa central (¿taponamiento cardiaco?)

En la fase intraoperatoria y ante la sospecha de una lesión grave ubicada al otro lado del diafragma, han sido también sugeridos ciertos recursos para confirmar dichas lesiones y motivar cambios en la táctica operatoria. En el transcurso de una laparotomía si surge la duda de una lesión cardiaca asociada, el uso de una ventana pericárdica por vía transdiafragmática podría confirmar la necesidad de agregar una toracotomía a la laparotomía. En el caso de estar realizando la intervención desde el tórax en ocasiones puede hallarse que la hemorragia era en realidad de origen abdominal y que estaba llegando al tórax a través de una laceración diafragmática.

En la presente serie de nuestra institución, en aquellos pacientes con TTAP que se presentaron normales hemodinámicamente o que alcanzaron rápidamente ese estado luego de la reanimación inicial y de la colocación de un drenaje pleural, no se evidenciaron errores en la secuencia elegida para el abordaje de sus cavidades afectadas así como una incidencia nula de mortalidad. En forma coincidente con lo reportado en la literatura en este grupo predominaron la heridas cortopunzantes y de ubicación en el lado izquierdo de la zona toracoabdominal. ${ }^{3,15}$ La condición hemodinámica de estos pacientes permitió en ellos la realización de TAC con contraste endovenoso en el caso de las HAF y de las toracoabdominales derechas, así como una videolaparoscopia diagnostica y eventualmente terapéutica en las heridas toracoabdominales izquierdas. $^{20}$

La mortalidad y el error en la secuencia de abordaje cavitario en las TTAP se presentó en esta serie exclusivamente en el segundo grupo, el de aquellos pacientes con anormalidad hemodinámica durante su asistencia. El análisis del mismo también mostró coincidencias con respecto a los reportes de la bibliografía. ${ }^{2,15} \mathrm{En}$ este segundo grupo la anormalidad hemodinámica por la condición de shock hipovolémico, el predominio del mecanismo de HAF y el error de secuencia de abordaje (factores todos ellos no presentes en el primer grupo) se asociaron a una mayor mortalidad.

El error de secuencia de abordaje se presentó en una cuarta parte de los pacientes de este segundo grupo (8/31) (25.8\%), en los cuales la cavidad abordada inicialmente no contenía la lesión más grave o aquella que resultaría letal. En cuatro de esos casos la cavidad abordada inicialmente había sido el tórax, y en la mitad restante el abdomen.

En el análisis de esos casos en los cuales inicialmente se abordó el tórax cuando la lesión más grave se hallaba en el abdomen, el error se relacionó más frecuentemente con un resultado falso de la ecografía, ya sea indicando liquido pericárdico que no estaba presente (falso positivo) u omitiendo liquido peritoneal que estaba presente (falso negativo). Estos errores fueron considerados potencialmente prevenibles, a diferencia del caso restante donde hubo un sangrado transdiafragmatico directo hacia el tórax desde una lesión de la vena cava retro hepática, no detectable ni clínicamente ni a través de la ecografía y por ello considerado un error no prevenible.

En el análisis de esos casos en los cuales inicialmente se abordó el abdomen cuando la lesión más grave se hallaba en el tórax, los errores se relacionaron en porcentajes iguales con dos situaciones: un sangrado transdiafragmatico directo hacia el abdomen desde una herida cardiaca sin taponamiento (error no prevenible) o una subestimación de un drenaje pleural con debito mayor a $1000 \mathrm{cc}$ (error prevenible). En estos últimos dos casos de débito hemático alto a través del drenaje pleural, lo cual evidenciaba una hemorragia severa en el tórax, también se registró una falta de radiografía de tórax luego de la colocación del avenamiento pleural. Esas placas radiográficas hubieran evidenciado, en una valoración retrospectiva en base a los hallazgos operatorios posteriores, a un hemotórax retenido importante y centinela de un sangrado activo intratorácico (Fig. 1).

Entre los 8 pacientes con error en la secuencia de abordaje fallecieron 7 (87.5\%), de los cuales en 6 casos la muerte se debió a hemorragia intraoperatoria. Si bien en estos casos se halló error en la secuencia operatoria y se podría pensar en un retraso para el control de ese sangrado, todos ellos presentaban un cuadro de shock hipovolémico secundario a una lesión cardiaca por HAF sin taponamiento o bien a una lesión de grandes vasos (aorta abdominal o vena cava inferior retrohepática), heridas las cuales ya de por si están afectadas por una mortalidad considerable. Esta circunstancia podría dificultar el análisis acerca de que grado de importancia tuvieron esos errores de manejo para influir en los fallecimientos.

Si bien esta serie de TTAP presenta limitaciones dadas por su número pequeño de casos y su índole retrospectiva, lo cual puede condicionar los resultados, consideramos que la experiencia aportada por el registro y análisis de estos errores de manejo puede ser valor en el objetivo de mejorar nuestra calidad de la asistencia en el futuro. Para dicho fin, esa experiencia recogida ha sido reflejada en un protocolo actual de nuestra institución para el manejo de los TTAP (Fig. 4).

TTAP: Traumatismos toracoabdominales penetrantes. Der: derecho. Izq: izquierdo. Toracotomia SR: Toracotomía de reanimación en shock room. HAF: herida por arma de fuego. HAB: herida cortopunzante. TAC: tomografía axial computada. VLP: videolaparoscopia diagnostica.

En este algoritmo, los pacientes en su ingreso son evaluados según las guías de la asistencia inicial de los traumatizados del curso ATLS. La ecografía extendida ha sido incorporada de modo sistemático en la revisión primaria dada su rapidez y factibilidad de aplicación en todas las emergencias. En aquellos pacientes con TTAP que ingresan con anormalidad hemodinámica se emplea el drenaje pleural en forma liberal y se monitorea cuidadosamente su débito, al mismo tiempo que se realiza su reanimación, un radiografía de tórax de control para descartar hemotórax retenido y la ecografía abdominal-pericárdica-pleural en busca de fuentes hemorrágicas. En casos extremos sin respuesta a la reanimación y con tensión arterial sistólica inferior a $70 \mathrm{~mm} \mathrm{Hg}$.y ausencia de pulso 


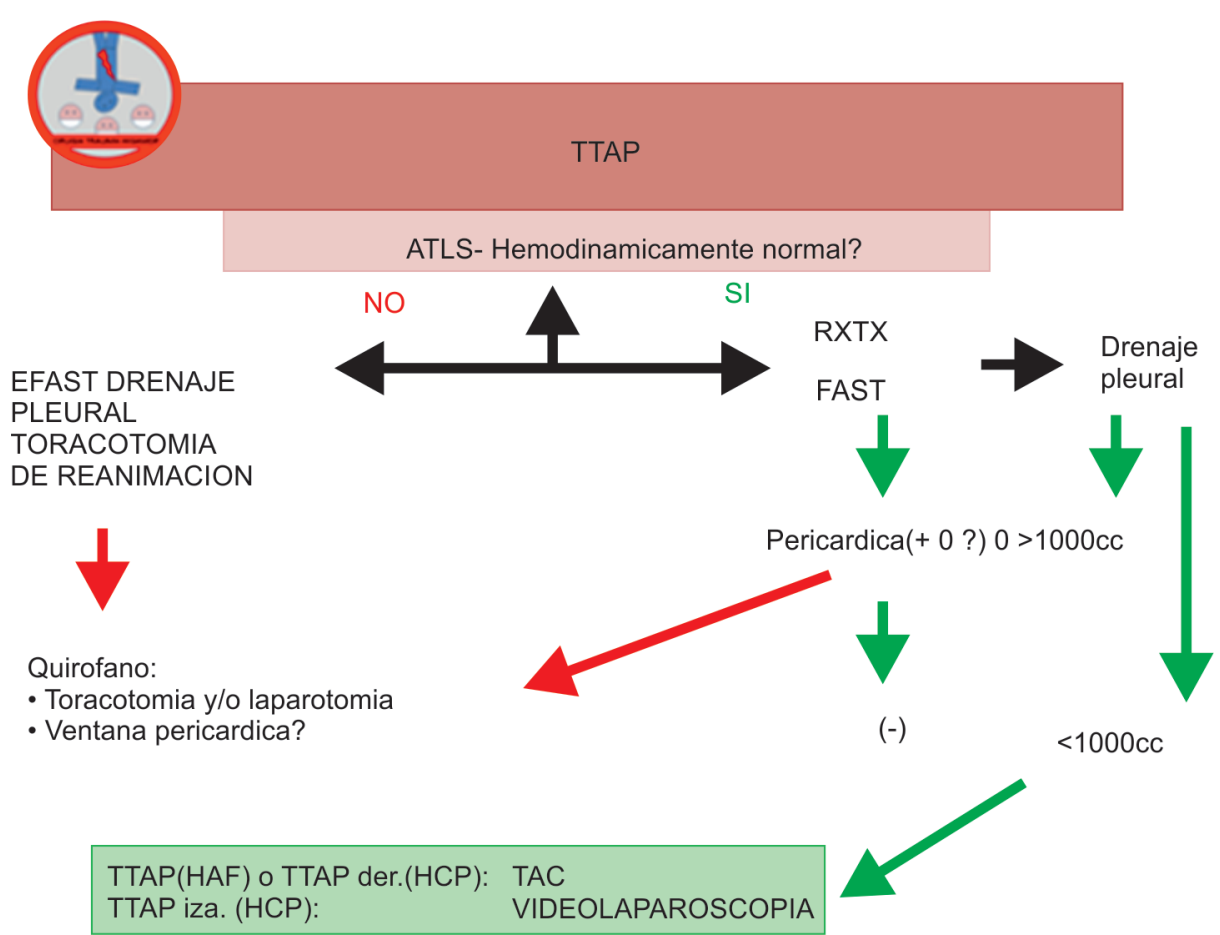

Fig. 4: Protocolo para el manejo de las heridas toracoabdominales penetrantes

radial, en los cuales hay condición de parada cardiaca inminente, se indica la toracotomía de reanimación, al igual que en aquellos pacientes que ingresan con parada cardiaca pero con signos vitales presenciados en la escena por el personal prehospitalario. Los pacientes descompensados son llevados a quirófano sin realizar otros estudios complementarios adicionales; en base a la ecografía previa y al débito del drenaje pleural se decide si tiene indicación de toracotomía y/o laparotomía, así como el orden en la secuencia de los procedimientos combinados.

En aquellos pacientes con TTAP que ingresan con normalidad hemodinámica, se realizan la ecografía extendida (abdominalpericárdica-pleural) y la radiografía de tórax como estudios iniciales. Si se diagnostica hemoneumotórax, se coloca drenaje pleural en la sala de shock y se monitorea cuidadosamente su débito, al mismo tiempo que se realiza una radiografía de tórax de control para descartar hemotorax retenido. Si el débito del drenaje pleural es superior a 1000 cc. de sangre o si la ecografía muestra liquido en el saco pericárdico, el paciente es trasladado a quirófano; en base a la valoración conjunta de la ecografía previa y del débito del drenaje pleural se decide si tiene indicación de toracotomía y/o laparotomía, así como el orden en la secuencia de los procedimientos combinados. Si bien en las normativas para el manejo inicial del traumatizado del curso ATLS se define el límite de 1500 cc para considerar a un hemotorax como masivo e indicar la toracotomía mayor, en la presente serie se consideró el umbral más bajo de 1000 cc para indicar la toracotomía mayor en pacientes compensados, en base a lo sugerido por otros autores. ${ }^{21,22}$

En algunos casos con presencia de líquido laminar en el saco pericárdico, la ventana pericárdica por vía transdiafragmatica o subxifoidea es un recurso a considerar ya en el quirófano para confirmar la presencia de hemopericardio. En los pacientes compensados, con ecografía pericárdica negativa y débito del drenaje pleural menor a 1000 cc se progresa hacia otros métodos complementarios específicos. Aquellos en esa condición y con HCP toracoabdominales izquierdas son llevados a quirófano para una videolaparoscopia diagnóstica con el objetivo de descartar lesiones diafragmáticas, entre otras. Los que presentan heridas toracoabdominales derechas o por HAF reciben una TAC con contraste endovenoso de modo de descartar distintas lesiones y de definir si tienen indicación de tratamiento operatorio o no operatorio (TNO). Al respecto, en las heridas toracoabdominales derechas existe una mayor posibilidad de un TNO, dada la presencia del hígado, que en el caso de las toracoabdominales izquierdas, donde son motivo de mayor preocupación las lesiones del diafragma o viscerales.

\section{Conclusione}

Los pacientes con TTAP y hemodinámicamente compensados presentaron predominio de HCP del lado izquierdo y no tuvieron errores en el manejo ni mortalidad. En aquellos con TTAP y shock, predominaron las HAF transfixiantes de la línea media, y este grupo fue el que presento los errores de manejo y la mortalidad de toda la serie. La mayoría de esos errores fueron considerados potencialmente prevenibles y se relacionaron con resultados falsos de la ecografía y con subestimación del débito del drenaje pleural y de la radiografía de tórax post-drenaje pleural.

\section{References}

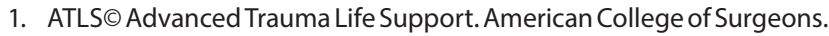
9 na edicion. 2012.

2. Clarke DL, Gall TMH, Thomson SR. Double jeopardy revisited: clinical decision making in unstable patients with, thoraco-abdominal stab woundsand, potential injuries in multiple body cavities. Injury 2011;42(5):478-481. DOI: 10.1016/j.injury.2010.06.027.

3. Asensio JA, Arroyo H, Veloz W, et al. Penetrating thoracoabdominal injuries: ongoing dilemma-which cavity and when? World J Surg 2002;26(5):539-543. DOI: 10.1007/s00268-001-0147-8. 
4. Menegozzo CAM, Damous SHB, Alves PHF, et al. "Pop in a scope": attempt to decrease the rate of unnecessary nontherapeutic laparotomies in hemodynamically stable patients with thoracoabdominal penetrating injuries. Surg Endosc 2020;34(1):261267. DOI: 10.1007/s00464-019-06761-7.

5. Koto ZM, Mosai F, Matsevych OY. The use of laparoscopy in managing penetrating thoracoabdominal injuries in Africa: 83 cases reviewed. World J Emerg Surg 2017;12(1):27. DOI: 10.1186/s13017-017-0137-2.

6. Uhlich R, Kerby JD, Bosarge $P$, et al. Diagnosis of diaphragm injuries using modern 256-slice CT scanners: too early to abandon operative exploration. Trauma Surg Acute Care Open 2018;3(1):e000251. DOI: 10.1136/tsaco-2018-000251.

7. Mjoli M, Oosthuizen G, Clarke D, et al. Laparoscopy in the diagnosis and repair of diaphragmatic injuries in left-sided penetrating thoracoabdominal trauma: laparoscopy in trauma. Surg Endosc 2015;29(3):747-752. DOI: 10.1007/s00464-014-3710-8.

8. Yücel M,ÖzpekA, Tolan HK, etal.Importance of diagnostic laparoscopy in the assessment of the diaphragm after left thoracoabdominal stab wound: a prospective cohort study. Ulus Travma Acil Cerrahi Derg 2017;23(2):107-111. DOI: 10.5505/tjtes.2016.91043.

9. Furák J, Athanassiadi K. Diaphragm and transdiaphragmatic injuries. JThorac Dis 2019;11(Suppl 2):S152-S157. DOI: 10.21037/jtd.2018.10.76.

10. Gao J, Du D, Li H, et al. Traumatic diaphragmatic rupture with combined thoracoabdominal injuries: difference between penetrating and blunt injuries. Chin J Traumatol 2015;18(1):21-26. DOI: 10.1016/j.cjtee.2014.07.001.

11. Prunty MC, Kudav S, Quick JA. Laparoscopic management of penetrating thoracoabdominal trauma. J Laparoendosc Adv Surg Tech A 2018;28(6):736-739. DOI: 10.1089/lap.2017.0482.
12. Gomez AC. Lesiones de diafragma. en de Torax Trauma. Vision Integral Para su Diagnostico y Tratamiento. Bogota: Editorial Medica Internacional; 2016. pp. 415-426.

13. Biff WL, Cioffi WG. Diafragma. en Feliciano D, Mattox K, Moore E. ., Trauma. 9na ed., Mc Graw Hill; 2021.

14. Mattox KL, Hirshberg A. Top Knife Allen MK, ed. 2005. pp. 149-151.

15. Hirshberg A, Wall MJ, Allen MK, et al. Double jeopardy: thoracoabdominal injuries requiring surgical intervention in both chest and abdomen. J Trauma 1995;39(2):225-231. DOI: 10.1097/00005373-199508000-00007.

16. Juan A, Traumatismo toracoabdominal. En: Trauma Prioridades. Cap 11-1.SAMCT. 2002. Ed Panamericana.

17. Matsushima K, Khor D, Berona K, et al. Double jeopardy in penetrating trauma: get FAST, get it right. World J Surg 2018;42(1):99-106. DOI: 10.1007/s00268-017-4162-9.

18. Quinn AC, Gibbons RC. What is the utility of the focused assessment with sonography in trauma (FAST) exam in penetrating torso trauma? Injury 2011;42(5):482-487. DOI: 10.1016/j.injury.2010.07.249.

19. Netherton S, Milenkovic V, Taylor M, et al. Diagnostic accuracy of eFAST in the trauma patient: a systematic review and meta-analysis. CJEM 2019;21(6):727-738. DOI: 10.1017/cem.2019.381.

20. Adolfo G, Alberto G, Sizenando VS. Trauma abdominal penetrante. en Trauma: Sociedad Panamericana de Trauma Ferrada R, Rodriguez A, ed. 2 da ed., Distribuna LTDA; 2009. pp. 338-347.

21. Wayne J. Meredith: traumatismo torácico: cuando y como intervenir. Surg Clin N Am 2007;87(1):95-118. DOI: 10.1016/j.suc.2006. 09.014 .

22. Carrillo E. Penetrating chest trauma. in: Initial management of injuries Sing R, Reilly P, ed. BMJ Books; 2001. pp. 87-95. 